\title{
A Multihop Cooperative Routing Algorithm for Minimizing the Number of Hops in Spectrum Sharing Networks
}

\author{
I-Te Lin and Iwao Sasase \\ Department of Information and Computer Science, Keio University \\ 3-14-1 Hiyoshi, Kohoku-ku, Yokohama-shi, Kanagawa-ken 223-8522, Japan \\ Email: itlin@sasase.ics.keio.ac.jp
}

\begin{abstract}
We propose a multihop cooperative routing (MCR) algorithm that extends the lengths of the $x$ axis projections of the hop distances and finds the multihop route that minimizes the number of hops in spectrum sharing networks. The cognitive relay and the cognitive receiver of each hop except the last one are selected by the following procedures. First, the cognitive node that is nearest away from the cognitive sender is selected as the cognitive relay, and let the cognitive destination (CD) be the cognitive receiver. Then, if the participation of the selected cognitive relay can not satisfy the QoS requirement of the cognitive transmission, among the cognitive receiver candidates that satisfy the QoS requirement of the cognitive transmission, the one whose $x$ coordinate has the largest difference from that of the cognitive relay is selected as the cognitive receiver. At the last hop, if the cognitive relay is available, the cooperative transmission is performed. Otherwise, the direct transmission is performed. Simulation results show that the MCR reduces the average number of hops compared to the FNR and outperforms the FNR in terms of the average end-to-end reliability, the average end-toend throughput, and the average required transmission power.
\end{abstract}

\section{INTRODUCTION}

In cognitive radio networks (CRNs), the cognitive user (CU) dynamically detects the unused spectrum holes released from the primary user (PU) and use them to transmit the data to improve the spectrum efficiency [1]. However, the requirement of the signal detection technique increases the implementation complexity of the CU. Besides, when the traffic load of the PU is heavy, very few unused spectrum holes are available [2].

Another way to implement the cognitive radio (CR) is to let the PU share the spectrum with the CU. In such network, the primary source (PS) and the cognitive source (CS) can transmit the data concurrently if the quality-of-service (QoS) requirements of the primary transmission and the cognitive transmission are both satisfied. The transmission power of the CS must be lower than a certain threshold in order to satisfy the QoS requirement of the primary transmission. Consequently, subject to the QoS requirement of the cognitive transmission, the CS has a maximum transmission distance. If the distance between the CS and the cognitive destination (CD) is longer than the maximum transmission distance of the $\mathrm{CS}$, the QoS requirement of the cognitive transmission can not be satisfied by using a single hop transmission. This problem can be solved by employing the multihop relaying.

In [2], the farthest neighbor routing (FNR) algorithm has been proposed to find a multihop route from the CS to the CD. First, the maximum transmission distance of the single hop cognitive transmission interfered by the PS is calculated. Then, among the nodes whose hop distances are less than or equal to the maximum transmission distance calculated in the last step, the node that is farthest away from the cognitive sender is selected as the cognitive receiver. The multihop route can be obtained by repeating the above two operations until the found node is the CD, or no node can be found.

The end-to-end reliability of the found multihop route is defined as the probability of successful reception of a packet at all cognitive receivers of all hops and can be given by

$$
\rho=\prod_{i=1}^{K} \beta_{c}^{(i)},
$$

where $K$ denotes the number of hops of the multihop route between the $\mathrm{CS}$ and the $\mathrm{CD}$, and $\beta_{c}^{(i)}$ represents the probability of successful reception of a packet at the cognitive receiver of the $i$ th hop. From (1), we can know that the end-to-end reliability degrades when the multihop relaying is used, and the degradation of the end-to-end reliability is significant when $K$ is large or any one of $\beta_{c}^{(i)}$, s is small.

To increase the end-to-end reliability, the number of hops should be reduced. As we know, the cooperative transmission is able to prolong the transmission distance. Therefore, combining the routing algorithm with the cooperative transmission can reduce the number of hops. Although the research of the cooperative routing in wireless networks has been done in [3]-[6], none of them address the issues of taking the interferences from other networks into account and minimizing the number of hops. Here, in the CRN where the cognitive sender and the cognitive relay have their own transmission power constraints and are interfered by the PS, we propose a multihop cooperative routing (MCR) algorithm that extends the lengths of the $x$ axis projections of the hop distances and finds the multihop route that minimizes the number of hops. The difference between the MCR and the FNR is that at each hop except the last one, in addition to the cognitive receiver, a cognitive relay is selected, and when the cognitive receivier 


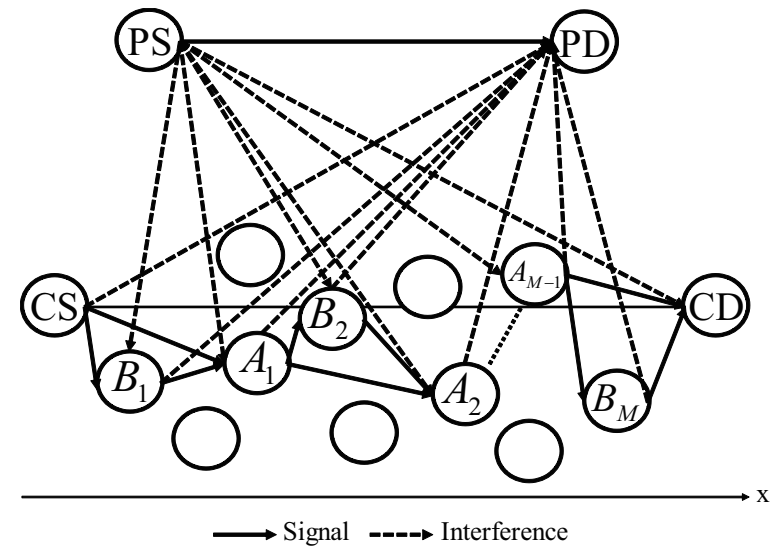

Fig. 1. The primary transmission coexists with the multihop cognitive cooperative transmission.

fails to decode the data transmitted from the cognitive sender, the selected cognitive relay retransmits the data overheard from the cognitive sender to the cognitive receiver. At the last hop, if the cognitive relay is available, the cooperative transmission is employed. Otherwise, the direct transmission is employed. Finally, we conduct the computer simulations to investigate the average numbers of hops of the FNR and the MCR and the performances of the FNR and the MCR in terms of the average end-to-end reliability, the average end-to-end throughput, and the average required transmission power.

\section{Multihop Cooperative Routing (MCR)}

\section{A. System Model}

We consider the network scenario illustrated in Fig. 1. There are a primary network (PN) and a $\mathrm{CRN}$ in the scenario. The PN consists of the PS and the primary destination (PD). The CRN consists of many cognitive nodes including the CS and the CD. The line between the CS and the CD is parallel to the $x$ axis. Let $M$ represent the number of hops of the multihop route $\left\{\mathrm{CS}, A_{1}, \ldots, A_{M-1}, \mathrm{CD}\right\}$. Let $A_{i}$ denote the cognitive receiver of the cognitive cooperative transmission performed in the $i$ th hop, where $i=1, \ldots, M-1$. Let $B_{j}$ represent the cognitive relay of the cognitive cooperative transmission performed in the $j$ th hop, where $j=1, \ldots, M$. We consider that the multihop relaying is performed without concurrency. Two or more than two cognitive cooperative transmissions will not occur simultaneously. As a result, at any time, the PD will be interfered by the cognitive sender or the cognitive relay of only one hop. Due to size and power constraints, we assume that each node is equipped with a single antenna. The channel of each link is characterized by the short term Rayleigh fading. We use the probability of successful reception of a packet as the QoS metric. If the signal-to-interference-plus-noise ratio (SINR) is higher than a certain predefined threshold, the receiver can receive the packet successfully. Under the Rayleigh fading channel, the author in [7] demonstrated that the probability of the successful reception of a packet can be

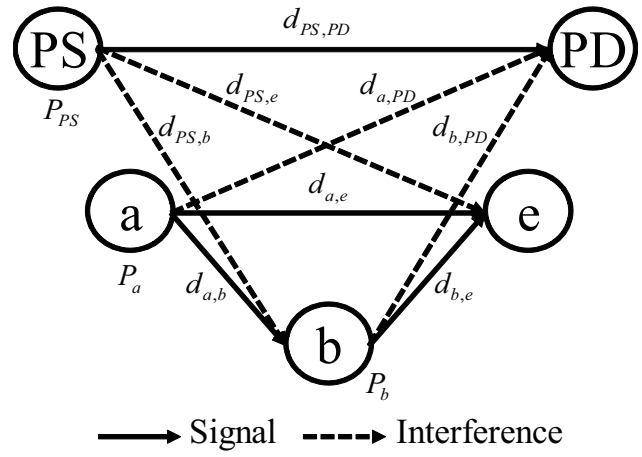

Fig. 2. The primary transmission coexists with the cognitive cooperative transmission.

written as

$$
\begin{aligned}
\beta & =\operatorname{Pr}(\operatorname{SINR} \geq \gamma) \\
& =\exp \left(-\frac{\gamma N_{0}}{P_{0} d_{0}^{-\alpha}}\right) \times \prod_{i=1}^{L} \frac{1}{1+\gamma \frac{P_{i}}{P_{0}}\left(\frac{d_{0}}{d_{i}}\right)^{\alpha}}
\end{aligned}
$$

where $\gamma$ denotes the SNIR threshold, $N_{0}$ represents the noise variance, $P_{0}$ is the sender transmission power, $d_{0}$ denotes the distance between the sender and the receiver, $\alpha$ represents the path loss exponent, $L$ is the number of interferers, $P_{i}$ denotes the transmission power of the $i$ th interferer, and $d_{i}$ represents the distance between the $i$ th interferer and the receiver.

\section{B. Cognitive Cooperative Transmission Interfered by the PS}

Figure 2 depicts the network scenario that the primary transmission coexists with the cognitive cooperative transmission. Node $a$, node $b$, and node $e$ denote the cognitive sender, the cognitive relay, and the cognitive receiver, respectively. We adopt the decode-and-forward incremental relaying protocol proposed in [8]. In the cooperative transmission, the transmission time is separated into two time slots. In the first time slot, the cognitive sender transmits the data to the cognitive receiver. Due to the broadcast nature of the wireless channel, the cognitive relay can also receive the data. Let $n_{i, j}$ denote the additive noise measured at node $j$ when node $i$ is the sender and can be modeled as zero mean complex Gaussian random variable with variance $N_{0}$. The received signal at the cognitive receiver and the cognitive relay in the first time slot can be expressed as

$$
y_{e, 1}=\sqrt{P_{a} d_{a, e}^{-\alpha}} h_{a, e} s_{a}+\sqrt{P_{P S} d_{P S, e}^{-\alpha}} h_{P S, e} s_{P S}+n_{a, e}
$$

and

$$
y_{b}=\sqrt{P_{a} d_{a, b}^{-\alpha}} h_{a, b} s_{a}+\sqrt{P_{P S} d_{P S, b}^{-\alpha}} h_{P S, b} s_{P S}+n_{a, b},
$$

respectively, where $P_{a}$ and $P_{P S}$ denote the transmission power of the cognitive sender and the PS, respectively, $d_{a, e}, d_{a, b}$, $d_{P S, e}$, and $d_{P S, b}$ represent the distances between the cognitive sender and the cognitive receiver, between the cognitive sender and the cognitive relay, between the PS and the cognitive receiver, and between the PS and the cognitive relay, respectively, $h_{a, e}, h_{a, b}, h_{P S, e}$, and $h_{P S, b}$ are the Rayleigh fading 
coefficients of the channels between the cognitive sender and the cognitive receiver, between the cognitive sender and the cognitive relay, between the PS and the cognitive receiver, and between the PS and the cognitive relay, respectively, and $s_{a}$ and $s_{P S}$ denote the data signals transmitted by the cognitive sender and the PS, respectively. The cognitive receiver decodes the data after the data is received. The decoding is correct if the SINR measured at the cognitive receiver is higher than a certain threshold. If the decoding is correct, the cognitive receiver informs the cognitive sender that the decoding is correct. Otherwise, the cognitive receiver informs the cognitive relay that the decoding is failed. Then, in the second time slot, the cognitive relay sends the data to the cognitive receiver if the decoding of the data received from the cognitive sender at the cognitive relay is correct. In the second time slot, the received signal at the cognitive receiver can be written as

$$
y_{e, 2}=\sqrt{P_{b} d_{b, e}^{-\alpha}} h_{b, e} s_{b}+\sqrt{P_{P S} d_{P S, e}^{-\alpha}} h_{P S, e} s_{P S}+n_{b, e},
$$

where $P_{b}$ denotes the transmission power of the cognitive relay, $d_{b, e}$ represents the distance between the cognitive relay and the cognitive receiver, $h_{b, e}$ is the Rayleigh fading coefficient of the channel between the cognitive relay and the cognitive receiver, and $s_{b}$ denotes the data signal transmitted by the cognitive relay.

C. Probability of the Successful Reception of a Packet of the Cognitive Receiver of the Cognitive Cooperative Transmission Interfered by the PS

By using the method in [9], we derive the probability of successful reception of a packet of the cognitive receiver of the cognitive cooperative transmission interfered by the PS. The probability of the failed reception of a packet of the cognitive receiver of the cognitive cooperative transmission interfered by the PS can be given by

$$
p_{e}^{F}=\operatorname{Pr}\left(\text { failure } \mid \operatorname{SINR}_{e, 1}<\gamma_{c}\right) \operatorname{Pr}\left(\operatorname{SINR}_{e, 1}<\gamma_{c}\right),
$$

where $\mathrm{SINR}_{e, 1}$ denotes the SINR measured at the cognitive receiver after the data transmitted from the cognitive sender is received at the cognitive receiver, and $\gamma_{c}$ represents the predefined SINR threshold of the cognitive transmission. The conditional failure probability in (6) can be expressed as

$$
\begin{aligned}
& \operatorname{Pr}\left(\text { failure } \mid \operatorname{SINR}_{e, 1}<\gamma_{c}\right)=\operatorname{Pr}\left(\operatorname{SINR}_{b}<\gamma_{c}\right) \\
& \quad+\operatorname{Pr}\left(\operatorname{SINR}_{b} \geq \gamma_{c}\right) \operatorname{Pr}\left(\operatorname{SINR}_{e, 2}<\gamma_{c} \mid \operatorname{SINR}_{e, 1}<\gamma_{c}\right),
\end{aligned}
$$

where $\operatorname{SINR}_{b}$ denotes the SINR measured at the cognitive relay after the data transmitted from the cognitive sender is received at the cognitive relay, and $\mathrm{SINR}_{e, 2}$ represents the SINR measured at the cognitive receiver after the data transmitted from the cognitive relay is received at the cognitive receiver. Because the event $\operatorname{SINR}_{e, 2}<\gamma_{c}$ and the event SINR $_{e, 1}<\gamma_{c}$ are independent, (7) can be rewritten as

$$
\begin{gathered}
\operatorname{Pr}\left(\text { failure } \mid \operatorname{SINR}_{e, 1}<\gamma_{c}\right)=\operatorname{Pr}\left(\operatorname{SINR}_{b}<\gamma_{c}\right) \\
+\operatorname{Pr}\left(\operatorname{SINR}_{b} \geq \gamma_{c}\right) \operatorname{Pr}\left(\operatorname{SINR}_{e, 2}<\gamma_{c}\right) .
\end{gathered}
$$

By substituting (8) into (6), we can obtain

$$
\begin{aligned}
p_{e}^{F}= & \operatorname{Pr}\left(\operatorname{SINR}_{b}<\gamma_{c}\right) \operatorname{Pr}\left(\operatorname{SINR}_{e, 1}<\gamma_{c}\right) \\
& +\operatorname{Pr}\left(\operatorname{SINR}_{b} \geq \gamma_{c}\right) \operatorname{Pr}\left(\operatorname{SINR}_{e, 2}<\gamma_{c}\right) \operatorname{Pr}\left(\operatorname{SINR}_{e, 1}<\gamma_{c}\right) .
\end{aligned}
$$

Let $p_{e}^{S}$ denote the probability of successful reception of a packet of the cognitive receiver of the cognitive cooperative transmission interfered by the PS. By substituting $p_{e}^{O}=1-p_{e}^{S}$ into (9) and using $\operatorname{Pr}(X<f)=1-\operatorname{Pr}(X \geq f)$, we can get

$$
\begin{aligned}
p_{e}^{S}= & \operatorname{Pr}\left(\operatorname{SINR}_{e, 1} \geq \gamma_{c}\right) \\
& +\operatorname{Pr}\left(\operatorname{SINR}_{b} \geq \gamma_{c}\right) \operatorname{Pr}\left(\operatorname{SINR}_{e, 2} \geq \gamma_{c}\right) \\
& -\operatorname{Pr}\left(\operatorname{SINR}_{b} \geq \gamma_{c}\right) \operatorname{Pr}\left(\operatorname{SINR}_{e, 2} \geq \gamma_{c}\right) \operatorname{Pr}\left(\operatorname{SINR}_{e, 1} \geq \gamma_{c}\right)
\end{aligned}
$$

where

$$
\begin{aligned}
& \operatorname{Pr}\left(\operatorname{SINR}_{e, 1} \geq \gamma_{c}\right)= \frac{\exp \left(-\frac{\gamma_{c} N_{0}}{P_{a} d_{a, e}^{-\alpha}}\right)}{1+\gamma_{c} \frac{P_{P S}}{P_{a}}\left(\frac{d_{a, e}}{d_{P S, e}}\right)^{\alpha}}, \\
& \operatorname{Pr}\left(\operatorname{SINR}_{b} \geq \gamma_{c}\right)=\frac{\exp \left(-\frac{\gamma_{c} N_{0}}{P_{a} d_{a, b}^{-\alpha}}\right)}{1+\gamma_{c} \frac{P_{P S}}{P_{a}}\left(\frac{d_{a, b}}{d_{P S, b}}\right)^{\alpha}},
\end{aligned}
$$

and

$$
\operatorname{Pr}\left(\operatorname{SINR}_{e, 2} \geq \gamma_{c}\right)=\frac{\exp \left(-\frac{\gamma_{c} N_{0}}{P_{b} d_{b, e}^{-\alpha}}\right)}{1+\gamma_{c} \frac{P_{P S}}{P_{b}}\left(\frac{d_{b, e}}{d_{P S, e}}\right)^{\alpha}} .
$$

\section{Routing Algorithm}

First, the cognitive node that is nearest away from the cognitive sender is selected as the cognitive relay. Let the cognitive receiver be the $\mathrm{CD}$. Check if the participation of the selected cognitive relay can satisfy the QoS requirement of the cognitive transmission $p_{e}^{S} \geq \delta_{c}$, where $\delta_{c}$ denotes the QoS threshold of the cognitive transmission. If so, the algorithm terminates. Otherwise, calculate the probability of the successful reception of a packet of each cognitive receiver candidate that is the cognitive node whose $x$ coordinate is larger than that of the cognitive relay. Then, among the cognitive receiver candidates that satisfy the QoS requirement of the cognitive transmission, the one whose $x$ coordinate has the largest difference from that of the cognitive relay is selected as the cognitive receiver. The multihop route can be established by repeating the procedures stated above until the selected cognitive receiver has the largest $x$ coordinate among all the cognitive nodes except the CD. The detail algorithm is presented as follows:

1) Set $i=0, j=1, k=0$, and $A_{0}=C S$.

2) Let $E_{g}$ denote the set of nodes whose $x$ coordinates are larger than the $x$ coordinate of node $g$ except the CD.

3) Among $E_{A_{i}}$, select the cognitive node that is nearest away from $A_{i}$ as the cognitive relay $B_{j}$.

4) Let $a=A_{i}, b=B_{j}$, and $e=C D$ and use (10) to calculate $p_{e}^{S}$. If $p_{e}^{S} \geq \delta_{c}$, or $B_{j}$ has the largest $x$ 
TABLE I

Simulation PARAMETERS

\begin{tabular}{ll}
\hline parameter & value \\
\hline $\begin{array}{l}\text { SINR thresholds } \gamma_{p} \text { and } \gamma_{c} \\
\text { noise power } N_{0}\end{array}$ & 3 \\
distances $d_{P S, P D}$ and $d_{C S, C D}$ & $100 \mathrm{dBm}$ \\
QoS threshold $\delta$ & 0.95 \\
$\begin{array}{l}\text { QoS thresholds } \delta_{p} \text { and } \delta_{c} \\
\text { number of the cognitive nodes } \\
\text { excluding the CS and the CD } \\
\text { transmission rate } R \\
\text { of the direct transmission }\end{array}$ & 0.9 \\
\end{tabular}

coordinate among all the cognitive node except the $\mathrm{CD}$, let $k=1$ and go to 7). Otherwise, go to the next step.

5) For each cognitive node that belongs to $E_{B_{j}}$, let $e$ be the cognitive node and use (10) to calculate $p_{e}^{S}$.

6) Among the cognitive nodes that belong to $E_{B_{j}}$ and have $p_{e}^{S}$ 's that are equal to or larger than $\delta_{c}$, select the one whose $x$ coordinate has the largest difference from that of $B_{j}$ as $A_{i+1}$. If $A_{i+1}$ has the largest $x$ coordinate among all the cognitive nodes except the $\mathrm{CD}$, let $k=1$.

7) If $k=0$, let $i=i+1, j=j+1$, and go to 3 ). Otherwise, terminate.

\section{Performance Evaluation}

The computer simulations are conducted to investigate the average numbers of hops of the FNR and the MCR and the performances of the FNR and the MCR in terms of the average end-to-end reliability, the average end-to-end throughput, and the average required transmission power. Table 1 lists the simulation parameters. The coordinates of the PS, the PD, the $\mathrm{CS}$, and the $\mathrm{CD}$ are $\left(0, d_{P S, C S}+50\right),\left(d_{P S, P D}, d_{P S, C S}+50\right)$, $\left(0, d_{C S, C D} / 2\right)$, and $\left(d_{C S, C D}, d_{C S, C D} / 2\right)$, respectively. The other cognitive nodes are randomly distributed in a square area. The coordinates of the vertices of the square are $(0,0)$, $\left(0, d_{C S, C D}\right),\left(d_{C S, C D}, 0\right)$, and $\left(d_{C S, C D}, d_{C S, C D}\right)$. The transmission power of the PS is given by

$$
P_{P S}=-\frac{\gamma_{p} N_{0}}{d_{P S, P D}^{-\alpha} \log \delta},
$$

where $\delta$ denotes the QoS threshold of the interference-free primary transmission. The simulation results are averaged over 10000 network scenarios.

Figure 3 shows the average number of hops versus the distance between the PS and the CS of the FNR and the MCR with $\alpha=3$ and $\alpha=4$. The observation from the average numbers of hops at different distances between the PS and the CS reveals that the average number of hops decreases or remains constant when the distance between the PS and the CS increases. The reason of the decrease of the average number of hops is explained as follows. When the distance between the PS and the CS becomes larger, the cognitive nodes suffer from less interference from the PS. As a result, the transmission power of the cognitive sender and the cognitive relay increases. Therefore, each hop distance of FNR and the

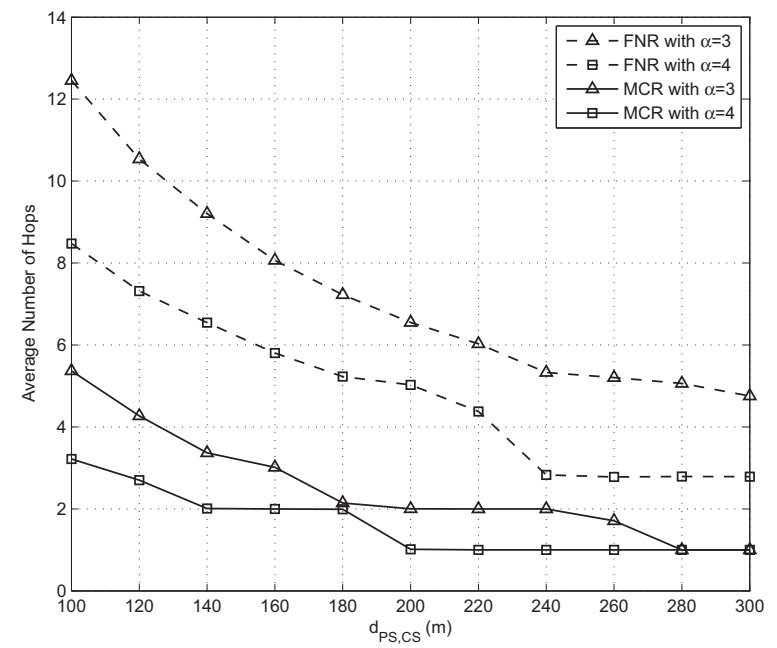

Fig. 3. Average number of hops versus the distance between the PS and the $\mathrm{CS}$ of the FNR and the MCR with $\alpha=3$ and $\alpha=4$.

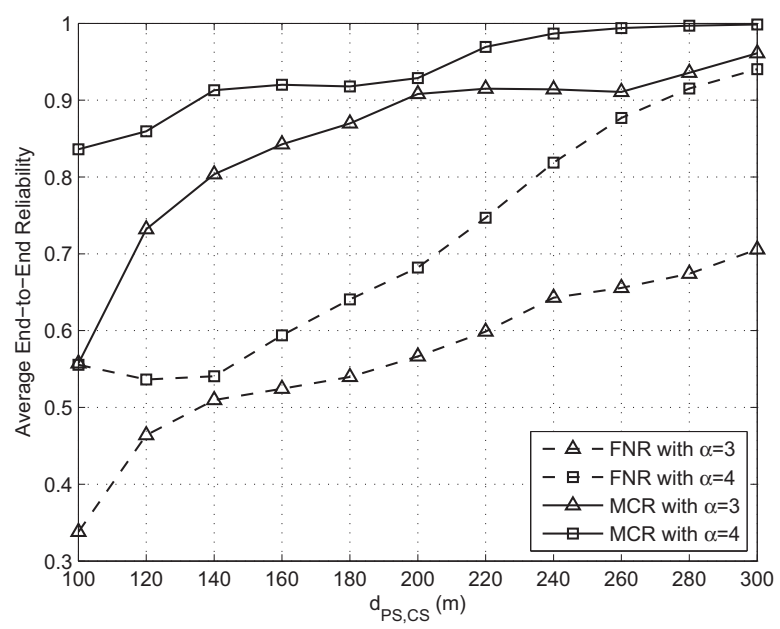

Fig. 4. Average end-to-end reliability versus the distance between the PS and the CS of the FNR and the MCR with $\alpha=3$ and $\alpha=4$.

lengths of the $x$ axis projections of the hop distances of MCR both become longer. The average numbers of hops are reduced due to the longer hop distances and the longer lengths of the $x$ axis projections of the hop distances. From Fig. 3, we can observe that at $\alpha=3$ and $\alpha=4$, the average number of hops of the MCR is less than that of the FNR. This phenomenon is because the cooperative transmission increases the lengths of the $x$ axis projections of the hop distances.

Figure 4 shows the average end-to-end reliability versus the distance between the PS and the CS of the FNR and the MCR with $\alpha=3$ and $\alpha=4$. From Fig. 4, we can observe that at $\alpha=3$ and $\alpha=4$, compared to the FNR, the MCR increases the average end-to-end reliability. This phenomenon is caused by that the MCR reduces the average number of hops compared to the FNR. 


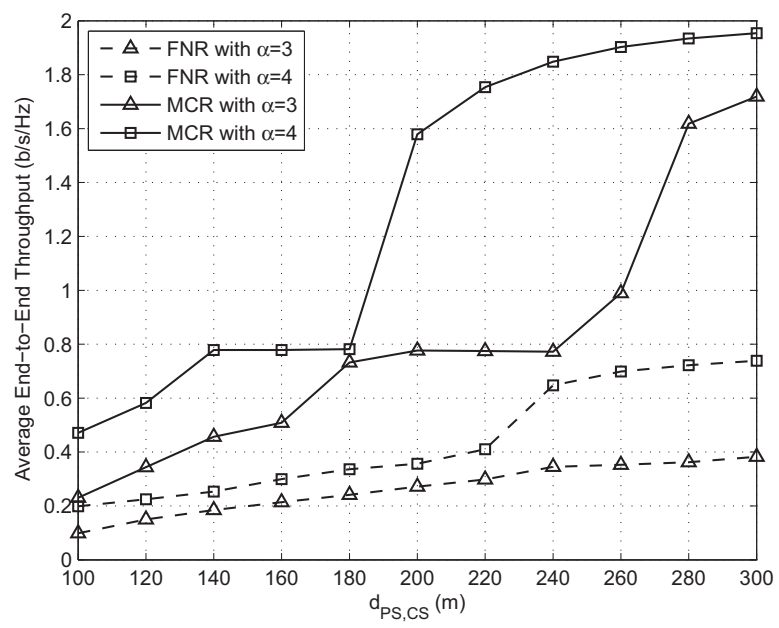

Fig. 5. Average end-to-end throughput versus the distance between the PS and the CS of the FNR and the MCR with $\alpha=3$ and $\alpha=4$.

Figure 5 shows the average end-to-end throughput versus the distance between the PS and the CS of the FNR and the MCR with $\alpha=3$ and $\alpha=4$. The throughput of the $i$ th hop, where $i=1, \ldots, M$, is defined as

$$
t_{c}^{(i)}=R^{(i)} \times \beta_{c}^{(i)},
$$

where $R^{(i)}$ denotes the transmission rate of the $i$ th hop. We know that the end-to-end throughput equals to the minimum of the throughputs of all hops. Besides, because the transmission occurs at only one hop at any time, the end-to-end throughput is reduced by the factor of $M$. The end-to-end throughput can be expressed as

$$
T=\min _{i=1, \ldots, M} \frac{t_{c}^{(i)}}{M} .
$$

From Fig. 5, we can observe that at $\alpha=3$ and $\alpha=4$, the MCR increases the average end-to-end throughput compared to the FNR. This phenomenon is also caused by that the MCR reduces the average number of hops compared to the FNR.

Figure 6 shows the average required transmission power versus the distance between the PS and the CS of the FNR and the MCR with $\alpha=3$ and $\alpha=4$. The required transmission power can be written as

$$
P=\sum_{i=1}^{M} P_{c}^{(i)},
$$

where $P_{c}^{(i)}$ represents the required cognitive transmission power of the $i$ th hop. From Fig. 6, we can observe that at $\alpha=3$ and $\alpha=4$, the required transmission power of MCR is less than that of the FNR.

\section{CONCLUSION}

In the FNR, the multihop relaying causes the large degradation of the end-to-end reliability. To reduce the number of hops, we have proposed a MCR algorithm that prolongs the lengths of the $x$ axis projections of the hop distances

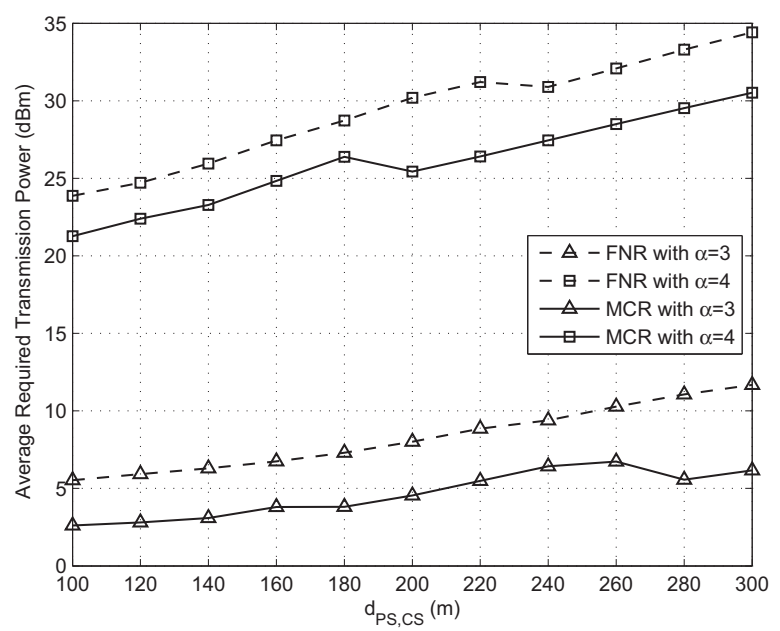

Fig. 6. Average required transmission power versus the distance between the PS and the CS of the FNR and the MCR with $\alpha=3$ and $\alpha=4$.

and finds the multihop route that minimizes the number of hops. Simulation results show that the average number of hops of the MCR is less than that of the FNR, and the MCR outperforms the FNR in terms of the average end-to-end reliability, the average end-to-end throughput, and the average required transmission power.

\section{ACKNOWLEDGMENT}

This work is partly supported by the Grant in the Global Center of Excellence Program "High-Level Global Cooperation for Leading-Edge Platform on Access Spaces" of Keio University from the MEXT, Japan.

\section{REFERENCES}

[1] J. Mitola III, "Cognitive radio: An integrated agent architecture for software defined radio." PhD thesis, Royal Institute of Technology (KHT), Stockholm, Sweden, May 2000.

[2] M. Xie, W. Zhang, and K.-K. Wong, "A geometric approach to improve spectrum efficiency for cognitive relay networks," IEEE Trans. Wireless Commun., vol. 9, no. 1, pp. 268-281, Jan. 2010.

[3] A. E. Khandani, J. Abounadi, E. Modiano, and L. Zheng, "Cooperative routing in static wireless networks," in Proc. 41st Annual Allerton Conference on Communication, Control, and Computing, pp. 2170-2179, Oct. 2003.

[4] F. Li, K. Wu, and A. Lippman, "Energy-efficient cooperative routing in multi-hop wireless ad hoc networks," in Proc. IEEE International Performance, Computing, and Communications Conference, pp. 215-222, Apr. 2006.

[5] C. Pandana, W. P. Siriwongpairat, T. Himsoon, and K. J. R. Liu, "Distributed cooperative routing algorithms for maximizing network lifetime," in Proc. IEEE Wireless Communications and Networking Conference, vol. 1, pp. 451-456, Apr. 2006.

[6] L. Ong and M. Motani, "Optimal routing for decode-and-forward based cooperation in wireless networks," in Proc. 4th Annual IEEE Communications Society Conference on Sensor, Mesh and Ad Hoc Communications and Networks, pp. 334-343, Jun. 2007.

[7] M. Haenggi, "On routing in random Rayleigh fading networks," IEEE Trans. Wireless Commun., vol. 4, no. 4, pp. 1553-1562, Jul. 2005.

[8] J. N. Laneman, D. N. C. Tse, and G. W. Wornell, "Cooperative diversity in wireless networks: efficient protocols and outage behavior," IEEE Trans. Inform. Theory, vol. 50, no. 12, pp. 3062-3080, Dec. 2004.

[9] A. K. Sadek, Z. Han, and K. J. R. Liu, "Distributed relay-assignment protocols for coverage expansion in cooperative wireless networks," IEEE Trans. Mob. Comput., vol. 9, no. 4, pp. 505-515, Apr. 2010. 